Research Paper

\title{
The Therapeutic Effect of Vitamin C in an Animal Model of Complex Regional Pain Syndrome Produced by Prolonged Hindpaw Ischemia-Reperfusion in Rats
}

\author{
Jae Hun Kim¹, Yong Chul Kim², Francis Sahngun Nahm³, Pyung Bok Lee ${ }^{3 凶}$ \\ 1. Department of Anesthesiology and Pain Medicine, Konkuk University Medical Center, Konkuk University School of Medicine; \\ 2. Department of Anesthesiology and Pain Medicine, Seoul National University Hospital; \\ 3. Department of Anesthesiology and Pain Medicine, Seoul National University Bundang Hospital.
}

$\triangle$ Corresponding author: Pyung Bok Lee, MD, PhD, Seoul National University Bundang Hospital, Department of Anesthesiology and Pain Medicine, Seoul National University Bundang Hospital, 82, Gumi-ro 173beon-gil, Bundang-gu, Seongnam-si, Gyeonggi-do, Korea. Phone: 82-31-787-7495; FAX: 82-31-787-4063; Email: painfree@snubh.org.

(c) Ivyspring International Publisher. This is an open access article distributed under the terms of the Creative Commons Attribution (CC BY-NC) license (https://creativecommons.org/licenses/by-nc/4.0/). See http://ivyspring.com/terms for full terms and conditions.

Received: 2016.09.23; Accepted: 2016.11.24; Published: 2017.01.15

\begin{abstract}
Objectives: It is known that increased free radicals from oxidative stress are one of the major causes of complex regional pain syndrome (CRPS). In this study, we tested the hypothesis that vitamin $C$ has a dose-related treatment effect in a chronic post-ischemic pain (CPIP) model.

Methods: A total of 49 male rats weighing 250 to $350 \mathrm{~g}$ were used. The 4 treatment groups were control (no medication), group 1.0 (administration of $1 \mathrm{mg} /$ day for vitamin $C$ for 5 days), group 2.5 (administration of $2.5 \mathrm{mg} /$ day vitamin $C$ for 5 days), and group 7.5 (administration of $7.5 \mathrm{mg} / \mathrm{day}$ vitamin $C$ for 5 days). The $50 \%$ mechanical withdrawal threshold and total blood antioxidant status (TAS) were measured before and after administration of vitamin C.

Results: Twenty-eight CPIP model rats were generated from 49 rats. Seven rats were randomly allocated to each group. The 50\% mechanical withdrawal threshold of group 2.5 (after the administration of vitamin $C$ ) was higher than that of the control group and group $1.0(P<0.05)$. At 1 day of the administration of vitamin $C$, the $50 \%$ mechanical withdrawal threshold of group 1.0 was higher than that of the control group and the blood levels of TAS in groups 2.5 and 7.5 were higher than that in control group $(P<0.05)$. Twelve days after the administration of vitamin $C$, the blood levels of TAS in groups 2.5 and 7.5 were lower than that of the control group $(P<0.05)$.

Discussion: The administration of a proper dose of vitamin $C$ can reduce oxidative stress, increase antioxidants, and recover the threshold for mechanical allodynia in the CPIP model.
\end{abstract}

Key words: antioxidants, chronic post-ischemic pain model, complex regional pain syndrome, oxidative stress, vitamin C.

\section{Introduction}

Complex regional pain syndrome (CRPS) is a disease that presents with severe pain.[1-3] The pathophysiology of CRPS is complex, and it is known that increased free radical production from oxidative stress is one of the major causes.[4-8]

The chronic post-ischemic pain (CPIP) model was introduced by Coderre et al.[9] in 2004. It is produced by prolonged ischemia and reperfusion of the hindpaw in rats. While there is no nerve damage, the rats do show color change, edema, hyperalgesia, and allodynia. Therefore, the model has been considered to be an animal model of CRPS Type 1.[9, 10] In studies of the CPIP model, several free radical scavengers were effective for the improvement of mechanical allodynia. $[9,11,12]$ However, there is no safety data for free radical scavengers in humans. Therefore, we hypothesized that if an easily available antioxidant such as vitamin C [13] is effective for the 
treatment of CRPS, it will be used for CRPS patients immediately.

Zollinger et al. [14-16] have reported that vitamin $C$ has a prophylactic effect in CRPS occurrence. The prophylactic effect of vitamin $C$ was thought to be related to the antioxidant effect. However, we did not find studies on the therapeutic effect of vitamin C in the CPIP model or in CRPS patients. In this study, we tested the hypothesis that vitamin $C$ has a therapeutic effect and that the effect shows a dose-response relationship in the CPIP model.

\section{Methods}

After approval by the Institutional animal care and use committee (52-2011-009) in our hospital, a total of 49 male-rats (Sprague-Dawley, Orient Bio Co., Ltd., Seoul, Korea) weighing 250-350 g were used. Rats were housed at two or three per cage with access to water and food. The room temperature remained constant at about $21^{\circ} \mathrm{C}$. The light and dark cycles were 12 hours. All rats had been acclimated to the laboratory environment over 7 days.

All experiments were conducted by one researcher. The CPIP models were produced by the methods introduced by Coderre et al.[9] After anesthesia with sevoflurane, ischemia was induced by a tight-fitting Nitrile 70 durometer O-ring (O-ring West, Seattle, WA) with a 7/32 inch internal diameter. This was placed for 3 hours on the left proximal ankle. Reperfusion was performed by removal of the ring lead. After confirmation of reperfusion for 30 minutes, the rats recovered from anesthesia. In order to confirm neurologic symptoms, the $50 \%$ withdrawal threshold was investigated using a von Frey filament (monofilament, Stoelting Co., Wood Dale, IL) for 48 hours (at 1 hour, 4 hours, 24 hours, and 48 hours after reperfusion) (Table 1). The rat was placed in a box with a wire grid bottom and was acclimated for 20 minutes. The von Frey filament was applied to the plantar skin of the hindpaw. The minimum stimulus intensity was $0.4 \mathrm{~g}$ and the maximum stimulus intensity was $15 \mathrm{~g}$. Each filament was applied 5 times. The interval of each stimulus was 3 minutes, and responses to 3 out of 5 stimuli were regarded as positive. We started the stimulus at $2.0 \mathrm{~g}$ and used a total of 6 filaments via the up-down method according to the response.[17] The 50\% withdrawal threshold was measured by the following formula

$50 \%$ withdrawal threshold $(\mathrm{g})=10[\mathrm{X}(\mathrm{f})+\mathrm{Kd}] / 10000$

$-X(f): \log$ unit of the last used von Frey filament.

-K: tabular value based on the pattern of + and response to stimuli presented by Dixon[17].

$-d$ : the mean difference in log units between applied stimuli. In this study, $d$ was 0.224 .

After confirmation of CPIP model, vitamin C was administered for 5 days to the oral cavity using a syringe before the morning meal. Three doses of vitamin $C$ were used or verifying the dose response relationship. These doses in rats were calculated based on the following human doses of vitamin C: 200 $\mathrm{mg} / 60 \mathrm{~kg}$ in humans is $1.0 \mathrm{mg} / 300 \mathrm{~g}$ in rats, 500 $\mathrm{mg} / 60 \mathrm{~kg}$ in humans is $2.5 \mathrm{mg} / 300 \mathrm{~g}$ in rats, and 1500 $\mathrm{mg} / 60 \mathrm{~kg}$ in humans is $7.5 \mathrm{mg} / 300 \mathrm{~g}$ in rats.

The rats were randomly allocated to 4 groups: control (no medication), group 1.0 (administration of $1 \mathrm{mg} / \mathrm{kg}$ vitamin $\mathrm{C}$ for 5 days), group 2.5 (administration of $2.5 \mathrm{mg} / \mathrm{kg}$ vitamin $\mathrm{C}$ for 5 days), and group 7.5 (administration of $7.5 \mathrm{mg} /$ day vitamin $C$ for 5 days). The $50 \%$ mechanical withdrawal threshold was measured before administration of vitamin $C$, on the $1^{\text {st }}$ and 5 th days of vitamin $C$ administration, and on the 7 th and 21st days after the discontinuation of vitamin C. Blood samples was obtained via the caudal vein of rats before the administration of vitamin $C$, on 1st and 5th days of the administration of vitamin $C$, and on 7 th day after the discontinuation of vitamin $\mathrm{C}$. Blood sampling was performed before the morning meal. Collected blood was placed in a tube with EDTA and stored at $8^{\circ} \mathrm{C}$ for 1 hour. Serum was obtained from the blood samples by centrifugation at $3000 \mathrm{rpm}$ at $4^{\circ} \mathrm{C}$. The serum was stored in polypropylene tubes at $-20^{\circ} \mathrm{C}$.

Table 1. Example of an experimental table in the $50 \%$ mechanical withdrawal threshold test.

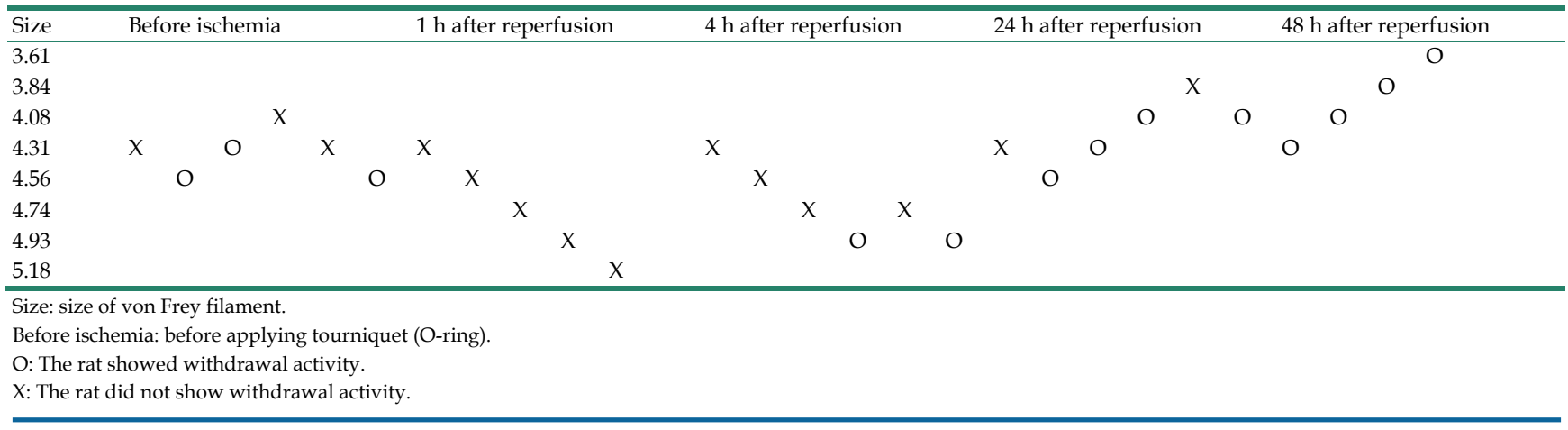


For evaluation of the effects of vitamin $C$, the $50 \%$ withdrawal threshold and total antioxidant status (TAS) were measured. These measurements were conducted on the 1st day and on the 5th day of the administration of vitamin $C$ and on the 7 th day after the discontinuation of vitamin $\mathrm{C}$.

TAS was measured from blood samples by using a TAS kit (Randox Labs., Crumlin, UK). The kit works based on plasma antioxidant substances inhibiting the oxidation of 2,2'-azino-di-[3-ethylbenzthiazoline-6-sul phonate] $\left(\mathrm{ABTS}^{\circledR}\right)$ by peroxidase and $\mathrm{H}_{2} \mathrm{O}_{2}$, which produces the radical cation $\mathrm{ABTS}^{\circledR+}$. The radical concentration was measured at $600 \mathrm{~nm}$ by spectrophotometry.

All statistical analyses were performed by SPSS version 18 (IBM, USA). The 50\% withdrawal threshold and TAS were analyzed by a repeated-measures ANOVA. In order to compare the control group and other groups at each period, the Mann-Whitney $U$ test was used. A $P$ value of $<0.05$ was considered statistically significant.

\section{Results}

Twenty-eight rats of the CPIP model were generated from 49 rats (Fig. 1). Seven rats were randomly allocated to each group. The 50\% mechanical withdrawal thresholds of group 2.5 after the administration of vitamin $C$ for 5 days were higher than that of the control group and group $1.0(P$ $<0.05$ ) (Fig. 2). At 1 day of the administration of vitamin C, the $50 \%$ mechanical withdrawal threshold of group $1.0(0.736 \pm 0.173 \mathrm{~g})$ was higher than that of the control group $(0.465 \pm 0.107, P=0.017)$. However, on the other vitamin $C$ administration days, there was no difference between the control group and group 1.0. There was no difference between the control group and group 7.5 or between group 2.5 and group 7.5 .

There was no difference in TAS blood levels between the control group and group 1.0. At 1 day of administration of vitamin $C$, the blood level of TAS in groups $2.5(1.133 \pm 0.053 \mathrm{mmol} / \mathrm{l})$ and $7.5(1.270 \pm$ $0.059 \mathrm{mmol} / \mathrm{l}$ ) were higher than that in control group $(0.958 \pm 0.246 \mathrm{mmol} / \mathrm{l}, P<0.05)$ (Fig. 3). At 5 days of administration of vitamin $C$, there was no difference between the control group and the other groups. Twelve days after the administration of vitamin $C$, the blood levels of TAS in groups $2.5(0.958 \pm 0.246$ $\mathrm{mmol} / \mathrm{l})$ and $7.5(0.841 \pm 0.344 \mathrm{mmol} / \mathrm{l})$ were lower than that in control group $(1.252 \pm 0.068 \mathrm{mmol} / \mathrm{l}, P<$ 0.05). Before administration of vitamin $C$, the blood level of TAS in group $7.5(1.199 \pm 0.083 \mathrm{mmol} / \mathrm{l})$ was higher than that in control group $(0.993 \pm 0.120$ $\mathrm{mmol} / \mathrm{l}, P=0.004)$.

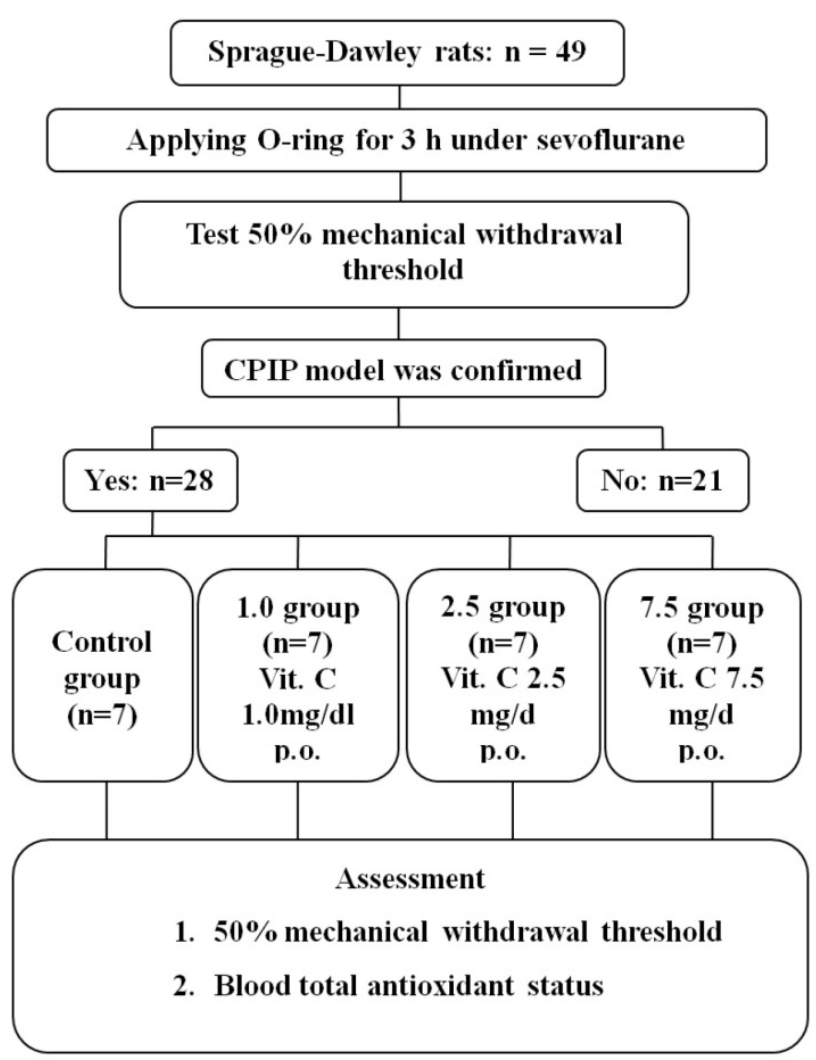

Figure 1. Flow chart of the study. Control group: no medication group. Group 1.0: administration of $1 \mathrm{mg} / \mathrm{kg}$ vitamin $C$ for 5 days. Group 2.5: administration of $2.5 \mathrm{mg} / \mathrm{kg}$ vitamin C for 5 days. Group 7.5: administration of $7.5 \mathrm{mg} /$ day vitamin C for 5 days.

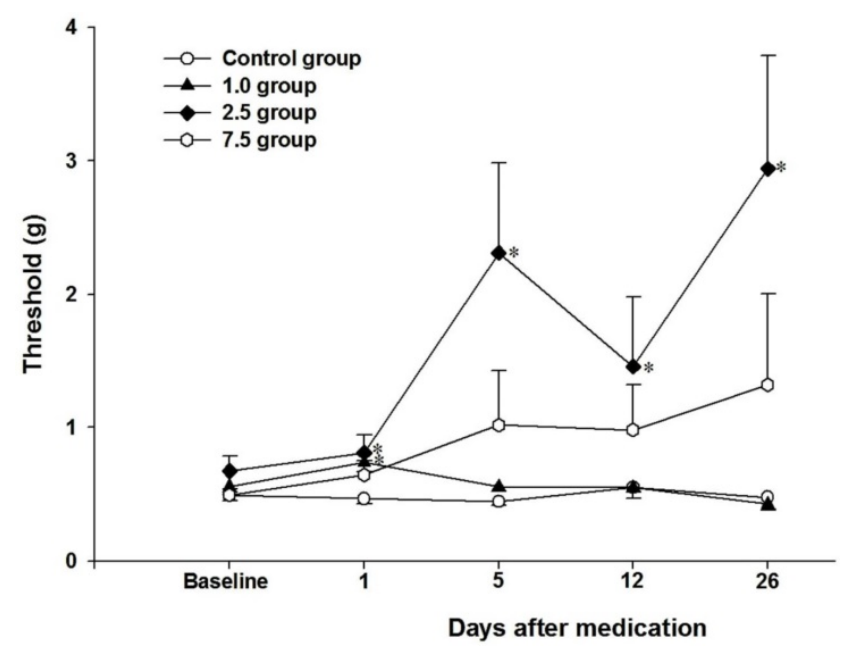

Figure 2. Time-course changes of the $50 \%$ mechanical withdrawal threshold within each group. Control group: no medication group. Group 1.0: administration of $1 \mathrm{mg} / \mathrm{kg}$ vitamin $C$ for 5 days. Group 2.5: administration of 2.5 $\mathrm{mg} / \mathrm{kg}$ vitamin $C$ for 5 days. Group 7.5: administration of $7.5 \mathrm{mg} /$ day vitamin $C$ for 5 days. $* P<0.05$ vs. control group. Error bar: standard error. 


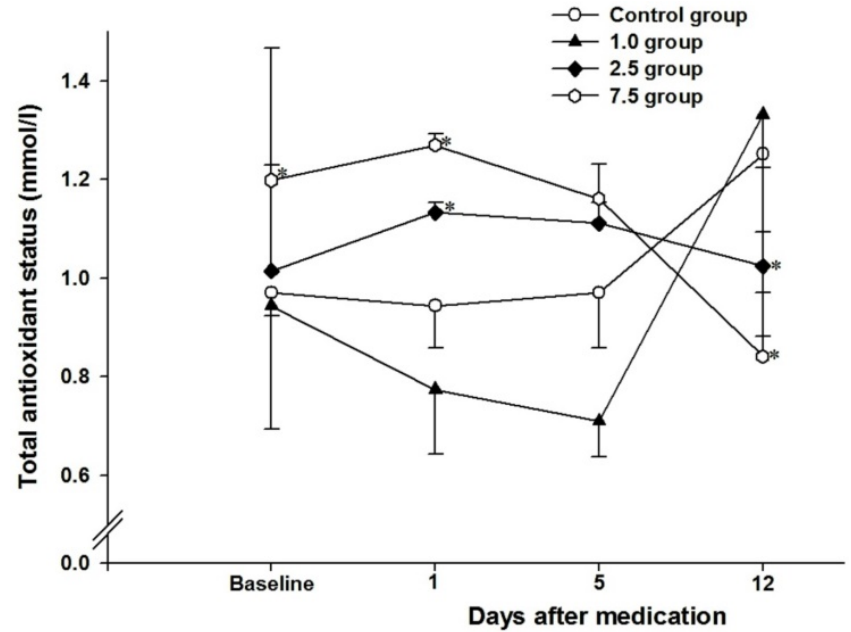

Figure 3. Time-course changes of blood total antioxidant status within each group. Control group: no medication group. Group 1.0: administration of 1 $\mathrm{mg} / \mathrm{kg}$ vitamin $C$ for 5 days. Group 2.5: administration of $2.5 \mathrm{mg} / \mathrm{kg}$ vitamin $C$ for 5 days. Group 7.5: administration of $7.5 \mathrm{mg} /$ day vitamin $C$ for 5 days. *: $P<0.05$ vs. control group. Error bar: standard error.

\section{Discussion}

In this study, the success rate of the CPIP model was about $57 \%$. Coderre at al. reported a success rate of approximately 70\%.[9] After the administration of vitamin $C$ for 5 days, the $50 \%$ withdrawal threshold in group 2.5 was higher than that in the control group and group 1.0. This indicates that administration of $2.5 \mathrm{mg}$ of vitamin $\mathrm{C}$ for 5 days can improve mechanical allodynia.

We also investigated the blood level of TAS for evaluating the correlation between improvement of mechanical allodynia and antioxidant status. The blood level of TAS in groups 2.5 and 7.5 at 1 day of administration of vitamin $C$ was higher than that in the control group, but the TAS level in groups 2.5 and 7.5 at 12 days after administration of vitamin $C$ was lower than that in the control group. At first, administration of vitamin $C$ in the $2.5 \mathrm{mg}$ and $7.5 \mathrm{mg}$ groups increased the level of TAS. The lower TAS level at 12 days after administration of vitamin C 2.5 $\mathrm{mg}$ and $7.5 \mathrm{mg}$ groups indicated lower oxidative stress in these groups than that in the control group. This indicates that administration of vitamin $C$ at doses of $2.5 \mathrm{mg}$ and $7.5 \mathrm{mg}$ might decrease the oxidative stress at 12 days after administration. In the $2.5 \mathrm{mg}$ group, this might have relevance to increased mechanical allodynia in the CPIP model. These results showed that administration of vitamin $C$ at $2.5 \mathrm{mg}$ could have a therapeutic effect on allodynia and that the improvement of oxidative stress could increase mechanical allodynia in the CPIP model.

We administered vitamin $C$ for 5 days in the early stage of the CPIP model and the improvement of mechanical allodynia lasted until at least 26 days (Fig.
2). At 1 day of administration of vitamin $C$, there was an increase in the $50 \%$ withdrawal threshold that was related to the dose-response relationship of vitamin $\mathrm{C}$ (no medication, $1 \mathrm{mg}$, and $2.5 \mathrm{mg}$ ). However, the $50 \%$ withdrawal threshold at other periods was no different between the control group and group 1.0. We hypothesized that the relatively low oxidative stress at the early stage might be controlled by $1.0 \mathrm{mg}$ of vitamin C and that the effect of an increased $50 \%$ withdrawal threshold was proportional to the dose of vitamin $C$ in the control group, group 1.0, and group 2.5. Nevertheless, at other periods, the relatively low dose of $1.0 \mathrm{mg}$ vitamin $\mathrm{C}$ might not decrease oxidative stress and could not increase the 50\% withdrawal threshold. On the contrary, in group 2.5, there were persistent improvements in the 50\% withdrawal threshold after vitamin $\mathrm{C}$ administration. Therefore, administration of $2.5 \mathrm{mg}$ vitamin $\mathrm{C}$ for 5 days could improve mechanical allodynia in CPIP models.

The pathophysiology of CRPS may be related to peripheral neuronal inflammation, local systemic inflammation, sympathetic nervous system dysfunction, and central nervous system abnormality.[2, 3, 18, 19] Several studies suggested that oxidative stress plays an important role in CRPS Type 1 pathogenesis. $[6,8,9,11,12,19,20]$ Neurogenic inflammation and chronic systemic inflammation can generate excessive free radicals.[5, 19, 20] The increased free radicals can increase vascular permeability and inflammatory reactions.[7, 21, 22] Free radical scavengers such as dimethyl sulfoxide, mannitol, and $\mathrm{N}$-acetylcysteine were effective for improvement of symptoms in CRPS patients.[23-27] Vitamin $C$ is a widely used dietary compound and well-known antioxidant.[13, 28] In previous studies, vitamin $C$ had prophylactic effects on the development of CRPS.[14-16] However, we cannot find an investigation of the treatment effects of vitamin C in the CPIP model or in CRPS patients. In our results, administration of vitamin $C$ can increase the antioxidant status and improve mechanical allodynia in the CPIP model. In this study, vitamin C was administered in the early stage of the CPIP model. Our results show the possibility of treatment effects of vitamin $C$ in the early stage of CRPS. Vitamin $C$ has been safely used for a long time. Therefore, the administration of vitamin C in CRPS patients can be easy and safe. Further evaluation of the treatment effect of vitamin $C$ and the dosage in CRPS patients will be necessary.

For the prophylactic effects of vitamin $C$ in humans, there was no difference between administration of vitamin $\mathrm{C}$ at $500 \mathrm{mg} /$ day or 1500 $\mathrm{mg} /$ day.[15] Therefore, the authors suggested a vitamin $C$ dose of $500 \mathrm{mg} /$ day. In this study, 
administration of vitamin $\mathrm{C}$ at $2.5 \mathrm{mg} /$ day was effective for the treatment of allodynia in the CPIP model. The dose of $2.5 \mathrm{mg}$ in rats was calculated from $500 \mathrm{mg}$ in humans. In humans, a daily dose over 100 mg causes urinary excretion of unmetabolized ascorbate and a daily dose over $500 \mathrm{mg}$ causes no increase in ascorbate concentration in tissue.[29] Therefore, $500 \mathrm{mg}$ of vitamin $\mathrm{C}$ can be a reasonable daily dose in humans.

There are some limitations in this study. First, the number of animals in each group was not large. Second, there was no statistical improvement in Group 7.5. We hypothesize that these results are related to the relatively low number of animals in each group. Third, the baseline TAS level in Group 7.5 was higher than in other groups. This might influence the results and be a source of bias in this study. It is impossible to statistically correct for different baselines in a non-parametric test. However, administration of vitamin $C$ at $2.5 \mathrm{mg}$ could increase TAS and improve mechanical allodynia in the CPIP model. This is a meaningful result in this study. Moreover, this result suggests the possibility of a therapeutic effect of vitamin C in early CRPS patients.

In conclusion, administration of vitamin $\mathrm{C}$ at 2.5 $\mathrm{mg}$ for 5 days could improve mechanical allodynia and increase the total antioxidant status during the administration periods. After the administration of 2.5 $\mathrm{mg}$ vitamin $\mathrm{C}$ for 5 days, TAS was decreased. This decrease in TAS was considered to be related to decreased antioxidant stress. The administration of a proper dose of vitamin $C$ can reduce oxidative stress, increase antioxidant levels, and recover the threshold for mechanical allodynia in the CPIP model. These results suggested the possibility of a therapeutic effect of vitamin C in early-stage CRPS.

\section{Acknowledgements}

This investigation was supported by Seoul National University Bundang Hospital.

\section{Competing Interests}

All authors reported no conflicts of interest.

\section{References}

1. Kang JE, Kim YC, Lee SC, Kim JH. Relationship between complex regional pain syndrome and working life: a Korean study. J Korean Med Sci. 2012; 27: 929-33.

2. Bruehl S. An update on the pathophysiology of complex regional pain syndrome. Anesthesiology. 2010; 113: 713-25.

3. van Eijs F, Stanton-Hicks M, Van Zundert J, Faber CG, Lubenow TR, Mekhail $\mathrm{N}$, et al. Evidence-based interventional pain medicine according to clinical diagnoses. 16. Complex regional pain syndrome. Pain Pract. 2011; 11: 70-87.

4. Kwak KH, Jung KY, Choi JY, Ryu T, Yeo JS, Park SS, et al. Contralateral allodynia and central change in the chronic post-ischemic pain model rats. Korean J Anesthesiol 2009; 56: 419-24.

5. Oyen WJ, Arntz IE, Claessens RM, Van der Meer JW, Corstens FH, Goris RJ. Reflex sympathetic dystrophy of the hand: an excessive inflammatory response? Pain. 1993; 55: 151-7.
6. Goris RJ, Dongen LM, Winters HA. Are toxic oxygen radicals involved in the pathogenesis of reflex sympathetic dystrophy? Free Radic Res Commun. 1987; 3: 13-8.

7. van der Laan L, Kapitein PJ, Oyen WJ, Verhofstad AA, Hendriks T, Goris RJ. A novel animal model to evaluate oxygen derived free radical damage in soft tissue. Free Radic Res. 1997; 26: 363-72.

8. van der Laan L, ter Laak HJ, Gabreels-Festen A, Gabreels F, Goris RJ. Complex regional pain syndrome type I (RSD): pathology of skeletal muscle and peripheral nerve. Neurology. 1998; 51: 20-5.

9. Coderre TJ, Xanthos DN, Francis L, Bennett GJ. Chronic post-ischemia pain (CPIP): a novel animal model of complex regional pain syndrome-type I (CRPS-I; reflex sympathetic dystrophy) produced by prolonged hindpaw ischemia and reperfusion in the rat. Pain. 2004; 112: 94-105.

10. Kim TK, Shin SW, Jung H, Lim DG. The effects of superoxide dismutase on the expression of c-fos gene in the chronic post-ischemic pain model rats. Korean J Anesthesiol 2008; 55: 78-86.

11. de Mos M, Laferriere A, Millecamps M, Pilkington M, Sturkenboom MC, Huygen FJ, et al. Role of NFkappaB in an animal model of complex regional pain syndrome-type I (CRPS-I). J Pain. 2009; 10: 1161-9.

12. Ryu TH, Jung KY, Ha MJ, Kwak KH, Lim DG, Hong JG. Superoxide and Nitric Oxide Involvement in Enhancing of N-methyl-D-aspartate Receptor-Mediated Central Sensitization in the Chronic Post-ischemia Pain Model. Korean J Pain. 2010; 23: 1-10.

13. Miftodei AM, Stefanache A, Spac A, Dorneanu V. Spectrometric determination of total antioxidant activity in chlorpromazine radical cation - ascorbic acid system. Rev Med Chir Soc Med Nat Iasi. 2013; 117: 806-11.

14. Zollinger PE, Tuinebreijer WE, Kreis RW, Breederveld RS. Effect of vitamin C on frequency of reflex sympathetic dystrophy in wrist fractures: a randomised trial. Lancet. 1999; 354: 2025-8.

15. Zollinger PE, Tuinebreijer WE, Breederveld RS, Kreis RW. Can vitamin C prevent complex regional pain syndrome in patients with wrist fractures? A randomized, controlled, multicenter dose-response study. J Bone Joint Surg Am. 2007; 89: 1424-31.

16. Zollinger PE, Kreis RW, van der Meulen HG, van der Elst M, Breederveld RS, Tuinebreijer WE. No Higher Risk of CRPS After External Fixation of Distal Radial Fractures - Subgroup Analysis Under Randomised Vitamin C Prophylaxis. Open Orthop J. 2010; 4: 71-5.

17. Chaplan SR, Bach FW, Pogrel JW, Chung JM, Yaksh TL. Quantitative assessment of tactile allodynia in the rat paw. J Neurosci Methods. 1994; 53: 55-63.

18. Moriwaki $\mathrm{K}$, Yuge $\mathrm{O}$, Tanaka H, Sasaki H, Izumi H, Kaneko K. Neuropathic pain and prolonged regional inflammation as two distinct symptomatological components in complex regional pain syndrome with patchy osteoporosis--a pilot study. Pain. 1997; 72: 277-82.

19. Taha R, Blaise GA. Update on the pathogenesis of complex regional pain syndrome: role of oxidative stress. Can J Anaesth. 2012; 59: 875-81.

20. Eisenberg E, Shtahl S, Geller R, Reznick AZ, Sharf O, Ravbinovich M, et al. Serum and salivary oxidative analysis in Complex Regional Pain Syndrome. Pain. 2008; 138: 226-32

21. Fraser PA. The role of free radical generation in increasing cerebrovascular permeability. Free Radic Biol Med. 2011; 51: 967-77.

22. Huet O, Dupic L, Harrois A, Duranteau J. Oxidative stress and endothelial dysfunction during sepsis. Front Biosci (Landmark Ed). 2011; 16: 1986-95.

23. Geertzen JH, de Bruijn $\mathrm{H}$, de Bruijn-Kofman AT, Arendzen JH. Reflex sympathetic dystrophy: early treatment and psychological aspects. Arch Phys Med Rehabil. 1994; 75: 442-6.

24. Zuurmond WW, Langendijk PN, Bezemer PD, Brink HE, de Lange JJ, van loenen AC. Treatment of acute reflex sympathetic dystrophy with DMSO 50\% in a fatty cream. Acta Anaesthesiol Scand. 1996; 40: 364-7.

25. Perez RS, Pragt E, Geurts J, Zuurmond WW, Patijn J, van Kleef M. Treatment of patients with complex regional pain syndrome type I with mannitol: a prospective, randomized, placebo-controlled, double-blinded study. J Pain. 2008; 9: 678-86.

26. Tan EC, Tacken MC, Groenewoud JM, van Goor H, Frolke JP. Mannitol as salvage treatment for Complex Regional Pain Syndrome Type I. Injury. 2010; 41: 955-9.

27. Perez RS, Zuurmond WW, Bezemer PD, Kuik DJ, van Loenen AC, de Lange JJ, et al. The treatment of complex regional pain syndrome type I with free radical scavengers: a randomized controlled study. Pain. 2003; 102: 297-307.

28. Halliwell B. Antioxidants in human health and disease. Annu Rev Nutr. 1996; 16: 33-50.

29. Zadak Z, Hyspler R, Ticha A, Hronek M, Fikrova P, Rathouska I, et al. Antioxidants and vitamins in clinical conditions. Physiol Res. 2009; 58 Suppl 1: S13-7. 